\title{
Turning Riboswitches Loose
}

\author{
Jörg S. Hartig*[a]
}

Riboswitches are mRNA-based modules that control gene expression through direct RNA-ligand interactions. ${ }^{\text {(1) }}$ In most cases, the regulatory ligands are metabolites that act on their own biosynthesis genes through feedback regulation. Since their discovery by Breaker and co-workers in 2002, nucleobases, amino acids, cofactors, as well as the amino sugar glucosamine-6-phosphate have been described to trigger changes in gene expression by binding to riboswitches. ${ }^{\text {(1) }}$ It became apparent that such protein-free regulation mechanisms are frequently found in bacteria with some examples such as thiamine pyrophosphate-dependent switches that even operate in plants and fungi. Riboswitches mostly operate through a common mechanism: ligand binding to an aptamer domain triggers a rearrangement in the $5^{\prime}$-untranslated region of an mRNA at which bacterial riboswitches usually are located. This event results in a structural rearrangement of the so-called expression platform, and provokes changes in transcription termination or initiation of translation. A different mechanism has been described in case of the glms-riboswitch in which glucosamine-6-phosphate acts as a cofactor and triggers scission of the respective mRNA through the action of a phosphodiestercleaving ribozyme.

The described riboswitches are typical examples of in cisacting regulatory mechanisms; this means that the riboswitch exclusively controls the expression of the riboswitch-containing mRNA. On the other hand, several examples of in transacting small regulatory RNAs (so-called SRNAs) have been described in bacteria. ${ }^{[2]}$ A subclass of sRNAs acts in trans through base-pairing to mRNAs that contain complementary sequences. In a recent report Cossart, Johansson and co-workers described for the first time that riboswitches formerly identified to be regulated by the cofactor S-adenosylmethionine (SAM) ${ }^{[3]}$ also act on other mRNAs in the pathogen Listeria monocytogenes. $^{[4]}$ L. monocytogenes is a Gram-positive bacterium that causes a food-borne infection called listeriosis. Over 40 different noncoding RNAs have been described in L. monocytogenes previously. ${ }^{[5]}$ Among them, SAM-dependent riboswitches are found in mRNAs that code for methionine and cysteine metabolism and uptake. They act by terminating transcription upon SAM binding, see Figure 1A. Surprisingly, two of these SAM riboswitches (SreA and $S r e B$ ) were found to influence the expression of several other genes. The influence on one of these genes, the virulence factor PrfA, was studied in more detail and found to be inhibited in trans, presumably through an an-

[a] Prof. Dr. J. S. Hartig

Department of Chemistry and Konstanz Research School Chemical Biology University of Konstanz

78457 Konstanz (Germany)

E-mail: joerg.hartig@uni-konstanz.de tisense mechanism. The authors demonstrated that the SAM riboswitch fragment originating from SAM-dependent transcription termination of SreA was able to hybridize to the target PrfA mRNA; this resulted in down-regulation of gene expression (see Figure 1B). SAM binding was not necessary for the inhibiting effect of the terminated riboswitch RNA on PrfA. Although the SreA riboswitch RNA and the PrfA MRNA were demonstrated to hybridize, the exact mechanism of how this interaction results in repression of PrfA is still unknown because the ribosome binding site located downstream of the hybridization site seems to be unaffected by this interaction. ${ }^{[4]}$

Interestingly, the PrfA mRNA is also regulated by an RNA thermometer that resides in its $5^{\prime}$-UTR. This element reduces gene expression by preventing the ribosome from binding to the mRNA at lower $\left(30^{\circ} \mathrm{C}\right)$ temperatures. At $37^{\circ} \mathrm{C}$, the RNA thermosensor allows for translation but then the PrfA mRNA is susceptible to in trans control through the action of the Sre riboswitch. ${ }^{[4]}$ In addition to transcriptional and post-translational

A)

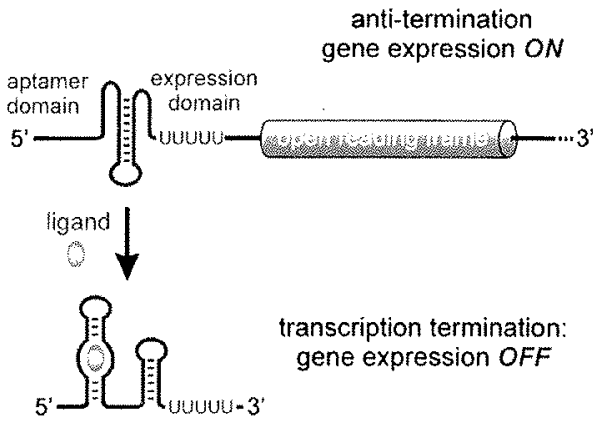

B)

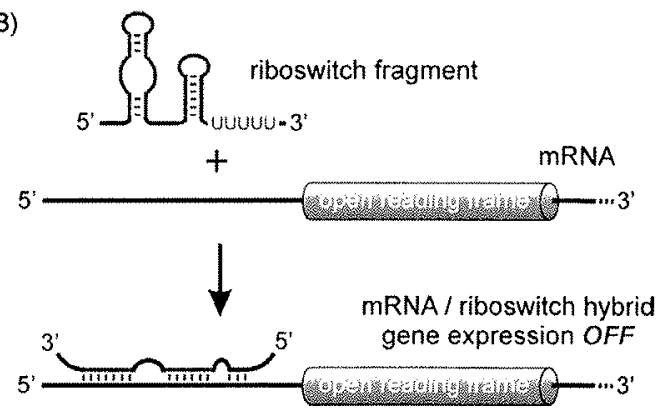

Figure 1. A riboswitch acting in trans. A) Riboswitches can act in cis through formation of an antiterminator stem, allowing for transcription of full-length mRNA. Ligand-binding to an aptamer-domain induces a terminator structure in the expression platform, resulting in premature termination of transcription and hence inhibition of gene expression. B) In L. monocytogenes, such terminated riboswitch fragments have been demonstrated to bind to other $m R N A s$, thereby regulating the expression in trans. 
control known for PrfA, it seems likely that the PrfA mRNA is regulated at least twice at the post-transcriptional level by RNA-based mechanisms: the virulence factor is only expressed efficiently at elevated temperatures and rich nutrients-optimum conditions for infection. On the other hand, the report by Loh et al. has not addressed the question whether SAM-dependent transcription termination of Sre sequences is necessary for the regulatory activity towards the PrfA message. Hence it is still unclear whether the intracellular SAM concentration affects PrfA expression because the full-length RNA containing the Sre elements could as well act in trans in a SAM-independent manner. In the latter case, the two distinct regulatory functions would just (coincidentally?) be harboured by the same piece of RNA.

Nevertheless, the example shows how nature makes use of very economic but sophisticated RNA-based mechanisms to implement complex regulatory functions. Along this line, the discovery of combinations of two regulatory elements in one message should be mentioned. For example, the Bacillus clausii metE mRNA contains both riboswitches for SAM as well as for coenzyme $B_{12}$; these riboswitches act in concert as a Boolean NOR gate. ${ }^{[6]}$ The discovery of complex RNA-based regulators in nature also has implications for the construction of artificial RNA-based switches. In recent years, several man-made RNA regulators have been described in the literature. ${ }^{[7,8]}$ So far, only cis-acting systems have been constructed in bacteria. The likelihood that trans-acting ligand-dependent RNA regulators occur in nature provides the opportunity to hard-wire synthetic RNA switches to have more than one output, and hence implement more complex behaviours by utilizing minimal information processing units made up from RNA. In order to realize such RNA synthetic biology tasks, further studies of the present as well as additional examples likely to exist in nature will be of great value.

\section{Acknowledgements}

I thank the VolkswagenStiftung, the Fonds der Chemischen Industrie and the University of Konstanz for continuing support.

Keywords, gene expression - post-transcriptional regulation . riboswitches - RNA - SAM

[1] A. Roth, R. R. Breaker, Annu. Rev. Biochem. 2009, 78, 305.

[2] J. Vogel, E. G. Wagner, Curr. Opin. Microbiol. 2007, 10, 262.

[3] W. C. Winkler, A. Nahvi, N. Sudarsan, J. E. Barrick, R. R. Breaker, Nat. Struct. Biol. 2003, 10, 701 .

[4] E. Loh, O. Dussurget, J. Gripenland, K. Vaitkevicius, T. Tiensuu, P. Mandin F. Repoila, C. Buchrieser, P. Cossart, J. Johansson, Cell 2009, 139, 770

[5] A. Toledo-Arana, O. Dussurget, G. Nikitas, N. Sesto, H. Guet-Revillet, D. Balestrino, E. Loh, J. Gripenland, T. Tiensuu, K. Vaitkevicius, M. Barthelemy, M. Vergassola, M. A. Nahori, G. Soubigou, B. Regnault, J. Y. Coppee, M. Lecuit, J. Johansson, P. Cossart, Nature 2009, 459, 950.

[6] N. Sudarsan, M. C. Hammond, K. F. Block, R. Welz, J. E. Barrick, A. Roth R. R. Breaker, Science 2006, 314, 300

[7] M. Wieland, J. S. Hartig, ChemBioChem 2008, 9, 1873.

[8] B. Suess, J. E. Weigand, RNA Biol. 2008, 5, 24 